\title{
LIDAR TO DETERMINE THE FRACTIONS OF ICE, LIQUID AND WATER VAPOR IN POLAR TROPOSPHERIC CLOUDS
}

\author{
Gilberto J. Fochesatto ${ }^{1}$, Oscar Galvez ${ }^{2}$, Pablo Ristori ${ }^{3}$, Douglas Keller ${ }^{1}$ and Enzo Fochesatto ${ }^{1}$ \\ ${ }^{1}$ Geophysical Institute, University of Alaska Fairbanks, AK 99775 USA \\ ${ }^{2}$ Laboratorio de Fisica de Materiales, UNED. Madrid, España. \\ ${ }^{3}$ Laboratorio Lidar. CITEDEF, Buenos Aires, Argentina. \\ *Email: gjfochesatto@alaska.edu
}

\begin{abstract}
A new Lidar combining Raman spectroscopy and linear polarization analysis is presented. This new instrument identifies the fraction of ice, liquid, and water vapor in low level polar tropospheric clouds and provides the polarimetric $\mathrm{S}$ and $\mathrm{P}$ state of the backscattering $532 \mathrm{~nm}$ Lidar signal. An overview of the research applications is given followed by a theoretical estimation of the Lidar returns. The instrument concept and optical characteristics are discussed. First Lidar profiles and instrument evaluations will be provided during the conference.
\end{abstract}

\section{INTRODUCTION}

The thermodynamic state of water in the troposphere plays a central role describing aerosol-cloud microphysical and dynamical processes in polar atmospheres. Spectrally resolved information on the thermodynamic phases of water in terms of the amount of ice, liquid, and vapor can be achieved by Laser spectroscopy (i.e., spontaneous Raman scattering). Such an instrument would be able to provide key information on specific cloud and aerosol processes and also account for changes in the surrounding environment as cloud and aerosol layers evolve and interact. Measuring the Raman shift up to $\sim 3800 \mathrm{~cm}^{-1}$ with spectral resolution better than $1 \mathrm{~cm}^{-1}$ allows selection of discrete Raman bands and vibrational lines to deduce the fractions of ice, liquid, and water vapor. Quantitative determination of concentration profiles requires knowing the Raman scattering cross section of the species under consideration. However, in this case this will be accomplished by referring all Raman returns to the well-known $\mathrm{N}_{2}$ cross section [1]. Interpretation of the vibrational levels of water vapor does not represent a critical issue. However, interpretation of the Raman spectra during phase transitions that occur in a super-cooled state requires some further research work. This has been documented in the past by divergences between $a b$ initio calculations, numerical approximations and laboratory experiments [2-8], and, of particular importance here, when cloud strata in the supercooled state undergo homogenous or heterogeneous nucleation $[9,10]$. In these cases, the intramolecular high frequency $\mathrm{OH}$ stretch mode shows significant changes around the isosbestic point in the spectral region 3000 to $3800 \mathrm{~cm}^{-1}$ as temperature decreases from ambient to $-24{ }^{\circ} \mathrm{C}$, as seen by the polarimetric sensitive Raman spectra [11]. And, as temperature lowers to $-35{ }^{\circ} \mathrm{C}$ and even lower, the asymmetric hydrogen bond $\left(<175 \mathrm{~cm}^{-1}\right)$ [12-15] is observed to decrease bond fluctuation, consistent with an increase in the formation of large structure polyhedral clusters in the region $<50 \mathrm{~cm}^{-1}[16]$. (see Figure 1).

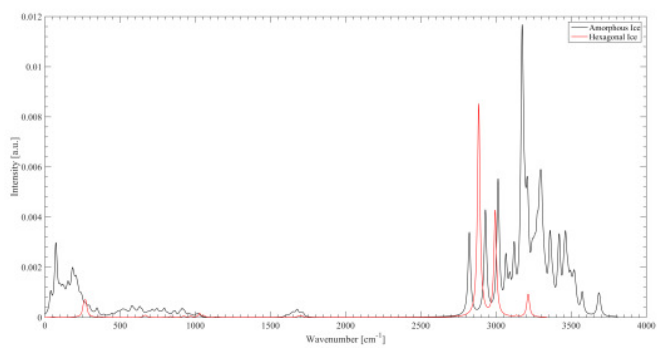

Figure 1. Theoretical Computation of Raman Spectrum of amorphous and hexagonal ice structures based on CASTEP. Lorentzian smearing of $20 \mathrm{~cm}^{-1}$ at $200 \mathrm{~K}$ and $10 \mathrm{~cm}^{-1}$ at $290 \mathrm{~K}$ respectively.

These complicated physicochemical processes that have been investigated by laser spectroscopy in laboratory settings are mature enough to be further studied in a Lidar configuration. This can provide more sophisticated aerosol-cloud freezing insight for model development. 
Furthermore, the spectroscopic information provided by this new Raman Lidar will be combined with the determinations of dynamic and microphysical structure of aerosols and cloud layers by means of polarimetric backscatter determination $[27,28]$. The simultaneous polarimetric-Raman capability will allow further identification of thermodynamic processes of water while in the presence of aerosols and clouds, identify non-spherical scatters, and detect ice formation, as well as estimate the crystal shape and habitat and their morphological changes, as they are also sensitive to the polarimetric observations from the laser line. Determination of atmospheric trace gas in the presence of aerosols is fundamental in studying physicochemical processes in multiphase and multicomponent atmospheric chemistry. These processes include aerosol seeding cloud formation, the role of aerosol surfaces assisting heterogeneous ice nucleation, and the chemical reactions on ice surfaces among others [17].

Since the inception of Raman Lidar methodology by Cooney [18], the technology has significantly evolved to a level that now allows simultaneous determination of several atmospheric chemical components using either Raman Lidars or combined Differential Absorption and Raman based Lidars to profile $\mathrm{N}_{2}, \mathrm{O}_{2}$ and $\mathrm{H}_{2} \mathrm{O}, \mathrm{SO}_{2}, \mathrm{O}_{3}$ and aerosols [19]. Most typical Raman Lidars [1, 20] involve the use of dedicated, custom made spectrometric units to spectrally resolve several molecular Raman features as a function of height. This currently available instrumentation operates in the visible and near UV spectra, normally stimulated by 532 and $355 \mathrm{~nm}$ pulsed lasers and in the UV-solar blind zone [19]. An example of the latter is the tropospheric ozone Lidar based on a $266 \mathrm{~nm}$ laser-pumped Raman multi-wavelength generation laser emission [21] and Raman Lidar using $266 \mathrm{~nm}$ as laser excitation [22].

On the other hand, Raman Lidars operating in the visible region are limited to nighttime minimal light conditions because the background solar radiation levels during the daytime surpass the Raman associated photon counting signal. In most of the cases, depending on the actual latitudinal location, the background radiation can top by several orders of magnitude the photon count level of the backscattering Raman signal. Some advances, including using Fabry-Perot etalons in the Lidar receiver, have partially overcome this issue [23]. Lidars operating in the UV solar blind region, in addition to being eye-safe, enhance the backscattering Raman cross section because of the short laser wavelength, typically $<300 \mathrm{~nm}$, but they experience strong signal absorption by ozone in the lower troposphere limiting their operation range [22]. Lidars operating in near-UV spectrum generally optimize the trade-off between maximizing the Raman cross section while minimizing background radiation. Still, they require the use of complicated optical arrangements, using multiple detectors and multichannel data acquisition receivers. A recent example [24] demonstrated the measurement of spectrally resolved water vapor and liquid Raman bands using a dispersive spectrometer coupled to a multichannel detector containing 32-fiber coupled integrated photomultipliers.

It is clear that in order to optimize the instrumental range -keeping the Raman cross section high enough while reducing background radiation- the laser stimulation has to be in nearUV spectrum. However, at shorter laser excitation wavelengths, Raman features narrow their spectral separation, making the receiver optics significantly more expensive. In this work, we therefore concentrate on demonstrating the feasibility of this Lidar spectroscopy, sacrificing signal-to-noise ratio and ranging capability by selecting the $532 \mathrm{~nm}$ wavelength as the laser excitation. Operating at this wavelength allows spectral requirements to be achieved within reasonable prices in the optics.

\section{LIDAR SIMULATION}

A simulation of the Raman Lidar returns, in MCPS ( $10^{6}$ counts per second), was performed for the main Raman lines to ensure detection limits, maximum vertical resolution, and minimum averaging time. The simulation was initialized by radiosonde profile assuming specific atmospheric properties and optical characteristics in the Lidar emission and receiver. This simulation allows for the evaluation of the signals to be expected in the Raman channels. The Lidar equation was implemented, including the terms of spectral extinction $\alpha\left[\mathrm{m}^{-1}\right]$, backscattering $\beta\left[\mathrm{sr}^{-1} \mathrm{~m}^{-1}\right]$ [25], and the Raman scattering cross sections of the main atmospheric features $\mathrm{N}_{2}, \mathrm{O}_{2}$ and $\mathrm{H}_{2} \mathrm{O}$ (see Table 1). The following expression shows a 
compact form of the Raman-Lidar equation as a function of the detected wavelength $(X)$.

$$
S_{X}(z)=\frac{E \cdot A}{z^{2}} \cdot \eta_{X} \cdot O(z) \cdot N_{X}(z) \cdot \sigma_{X}^{R A M}\left(\lambda^{R A Y}, \lambda_{X}^{R A M}\right) \cdot \operatorname{Tr}\left(z, \lambda^{R A Y}\right) \cdot \operatorname{Tr}\left(z, \lambda_{X}^{R A M}\right)
$$

$\mathrm{E}$ is the laser energy per pulse, $\mathrm{A}$ is the telescope effective collecting area, $\mathrm{z}$ is the height, $\eta_{X}$ is the optical throughput at the Raman wavelength $\left(\lambda_{X}^{R A M}\right), O(z)$ is the overlapping Lidar function, $N_{X}(z)$ is the concentration profile of the species of interest, $\sigma_{X}^{R A M}\left(\lambda^{R A Y}, \lambda_{X}^{R A M}\right)$ is the Raman cross section of the species of interest depending on the laser stimulation $\lambda^{R A Y}$ and the Raman wavelength shift $\lambda_{X}^{R A M}, \operatorname{Tr}\left(z, \lambda^{R A Y}\right)$ is the Rayleigh transmission of the atmosphere, including molecules and aerosols, at the laser wavelength, and $\operatorname{Tr}\left(z, \lambda_{X}^{R A M}\right)$ is the atmospheric transmission at the Raman wavelength in the time of flight returning to the telescope.

In this case, uniform aerosol vertical concentration profile in the atmospheric boundary layer (ABL) and wavelength scaling was considered, giving a specific Angstrom coefficient to simulate the spectral extinction due to aerosols [26]. The Lidar signal computation includes a random noise signal level based on a Poisson distribution to simulate real noise photondetection conditions and the spectral gain curve associated with the detector. The MCPS photon count rate is given at the entrance of the spectroscopic device. The optical throughput of the device that produces the spectroscopy of the backscattered signal strongly changes depending on the spectral characteristics of the analyzer (e.g., dichroic mirrors and filters). The laser emission and receiver parameters, including the detector characteristics, are indicated in Table 1. Figure 2 illustrates the MCPS simulation of the molecular Raman channels. Additionally, theoretical ab initio calculations of Raman spectra based on hexagonal microphysical ice structure and amorphous ice consisting of 20 water molecules (for sub-freezing temperatures) and the theoretical Raman spectrum of amorphous ice consisting of 20 water molecules is shown in Fig. 1. This information, combined with that in Fig. 3, suggests that strong changes in a band around $3100 \mathrm{~cm}^{-1}$ will indicate the formation and presence of ice, while liquid content should be determined after the isosbestic point where ice features are not prominent $\sim 3370 \mathrm{~cm}^{-1}$.
Table 1. $532 \mathrm{~nm}$ - Raman Lidar Simulation

\begin{tabular}{ll}
\hline \multicolumn{2}{l}{ Laser Emission Surelite SL II 10 Continuum Lasers } \\
\hline Wavelength & $1064.0 / 532 \mathrm{~mm}$ \\
Linewidth & $1.0 \mathrm{~cm}^{-1}$ \\
Pulse repetition frequency & $10 \mathrm{~Hz}$ \\
Energy per pulse & $650 / 300 \mathrm{~mJ}$ \\
Lidar Receiver & \\
Smith-Cassegrain & $\mathrm{F} / 5$ \\
Primary mirror diameter & $50 \mathrm{~cm}$ \\
Focal & $2.5 \mathrm{~m}$ \\
Custom Spectrometer & $\mathrm{F} / 5 \mathrm{input}$ \\
Dichroic / Razor filters based & Adaptor to F/2.5 coupled to fiber \\
Optics and Fiber Optic coupler & $\mathrm{NA}=0.2-1 \mathrm{~mm}$ core \\
& \\
Detection & $\mathrm{H} 10720-20$ \\
Hamamatsu PMT & $630 \mathrm{~nm}$ \\
Peak wavelength & $78 \mathrm{~mA} / \mathrm{W}$ \\
Cathode Radiant Sensitivity & 0.02 \\
Cathode Quantum Efficiency & $10^{6}$ \\
Detector Gain & $1 \mathrm{~min}$. \\
Accumulation time & $1.5 \mathrm{~m}$ \\
Vertical resolution & $607 \mathrm{~nm} \mathrm{~N}-2331 \mathrm{~cm}^{-1}$ \\
Angle tuning Raman features & $643 \mathrm{ice}-3175.3 \mathrm{~cm}^{-1}$ \\
& $650 \mathrm{liquid}-3373.6 \mathrm{~cm}^{-1}$ \\
& $660 \mathrm{~nm} \mathrm{H} \mathrm{O}^{-1}-3651 \mathrm{~cm}^{-1}$ \\
\hline
\end{tabular}

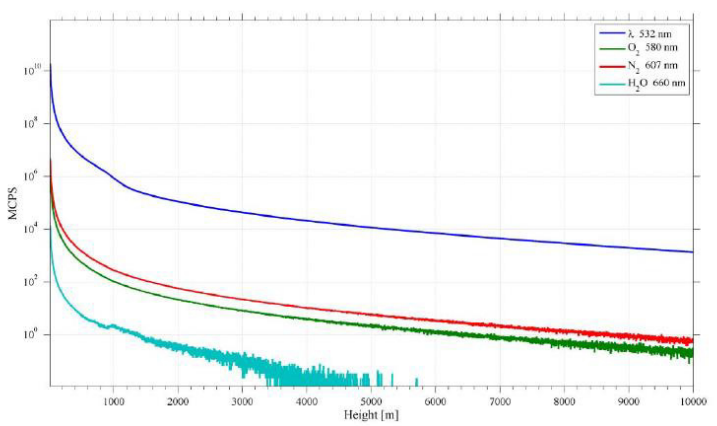

Figure 2. Raman Lidar Simulation. MCPS for 532 $\mathrm{nm}$ and Raman lines: $\mathrm{O}_{2}$ at $580 \mathrm{~nm} ; \mathrm{N}_{2}$ at $607 \mathrm{~nm}$ and $\mathrm{H}_{2} \mathrm{O}$ at $660 \mathrm{~nm}$. Thermodynamic profile from the NWS-PAFA June 21, 2006 at OOUTC (3 PM$A K T)$. ABL height was at $1 \mathrm{~km}$, entrainment zone thickness of $200 \mathrm{~m}$ and ozone concentration set to $40 \mathrm{ppb}$ both $A B L$ and free troposphere. Aerosol optical thickness at $380 \mathrm{~nm}$ was 0.3 , scaling wavelength Angstrom coefficient was 1.3 and a backscatter to extinction ratio of $0.012 \mathrm{sr}^{-1}$.

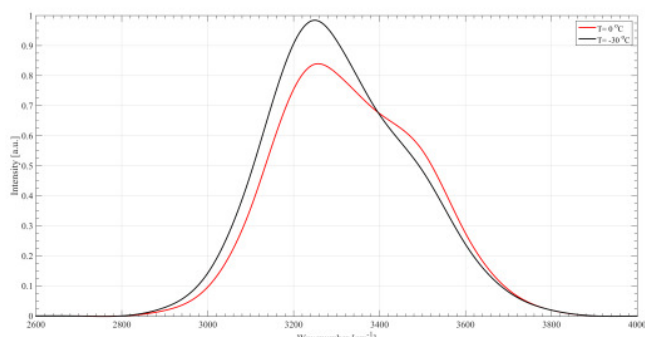

Figure 3. Raman Spectra of Water clusters, adapted from [11].

\section{INSTRUMENT DESIGN}

The Lidar emitter is based on a frequency doubled $\mathrm{Nd}$ :YAG pulsed laser operating at $532 \mathrm{~nm}$. The Lidar receiver is based on a Smith-Cassegrain F/5 with a $0.5 \mathrm{~m}$ primary mirror diameter with 
optimized optical throughput for visible spectroscopy. In the receiver, after splitting the linear polarization modes ( $\mathrm{S}$ and $\mathrm{P}$ ), the telescope is fiber coupled to a spectrometer designed to separate the vibrational Raman lines corresponding to $\mathrm{N}_{2}$ and $\mathrm{H}_{2} \mathrm{O}$, and the Raman features corresponding to Raman bands of ice at $3175.3 \mathrm{~cm}^{-1}$ and liquid at $3373.6 \mathrm{~cm}^{-1}$.

The instrument layout is shown in Figure 4.

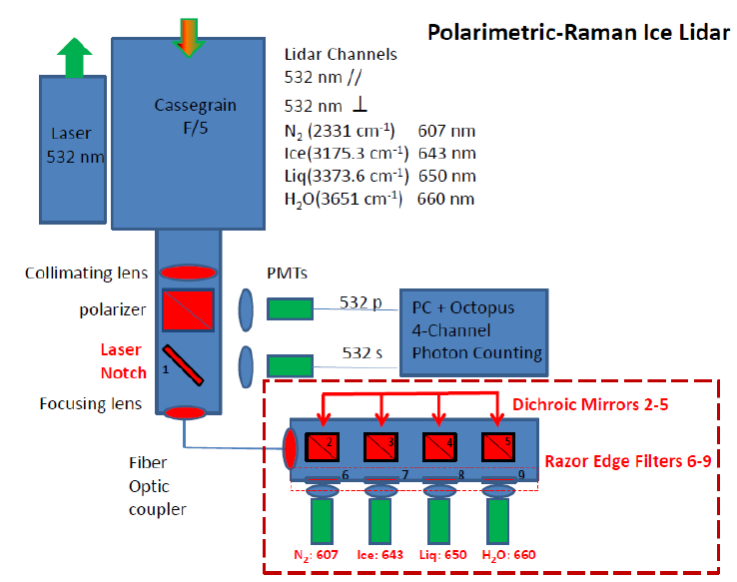

Figure 4. Polarimetric-Raman Ice Lidar Optical Layout.

\section{CONCLUSIONS}

A new Lidar system is being developed to investigate aerosol-cloud processes and composition in polar atmospheres. In this presentation a demonstration of feasibility will be presented illustrating several case examples in high latitude polar atmosphere.

\section{ACKNOWLEDGEMENT}

This project was funded by the US-NSF AGS1443222. The authors recognize funding from the EU 2013-program Advanced Spectroscopy in Chemistry, Complutense University of Madrid and fruitful discussions with Prof. Rafael Escribano CSIC, Madrid, Spain.

\section{REFERENCES}

[1] Turner D. D. and D. N. Whiteman. 2002. Handbook of Vibrational Spectroscopy. Edited by J. M. Chalmers and P. R. Griffiths. John Wiley and Sons Ltd, Chichester, 2002.

[2] Sceats M., S. A. Rice and J.E. Butler. 1975.

J. Chem. Phys., 63, 12, 5390-5400.

[3] Irish D., T. Jary and C. Ratcliffe. 1982.

Applied Spectroscopy, 36, 2, 137-140.
[4] Ratcliffe C. and D. Irish. 1982. J. Phys. Chem. 86, 4897-4905.

[5] Yeh Y., J.H. Bilgram and W. Kanzig. 1982.

J. Chem. Phys., 77(5), 2317-2321.

[6] Monosmith W. and G. Walrafen. 1984. Chem.

Phys. 81, 2, 669-674.

[7] Lee Ch., et al. 1993. Phys. Rev. B, 47, 9, 48634872.

[8] Walrafen G. 2006. J. Chem. Phys., 124,

184505; doi: 10.1063/1.2188942

[9] Angel C.A. Ann. Rev. Phys. Chem. 1983.34: 593-630

[10] Sokolov A., J. Hurst and D. Quitmann. 1995. Physical Review B., 51, 18, 12865-12868.

[11] D'Arrigo G. et al. 1981. J. Chem. Phys. 75: 4264-4270.

[12] Krishnamurthy S., et al. 1983. J. Chem. Phys. 79, 12, 5863-5870.

[13] Kanno H., K. Tomikawa and O. Mishima.

1998. Chem. Phys. Lett., 293, 412-416.

[14] Johari G. and O. Andersson. 2007.

Thermochimica Acta, 461, 14-43.

[15] Suzuki H., et al., 2012. J. Chem. Phys., 136, 234508; doi: 10.1063/1.4729476.

[16] Hare D.E. and S.M. Sorensen. 1990. J. Chem. Phys., 93, 1, 25:33.

[17] Hoose C. and O. Möhler. 2012. Atmos.

Chem. Phys., 12, 9817-9854.

[18] Cooney J. 1969. J. Appl. Meteor. 9, 182-184.

[19] Weitkamp C. 2005. Springer. pp 455.

[20] Di Girolamo P. et al. 2004. Geophys. Res.

Lett. 31, L01106, doi:10.1029/2003GL018342.

[21] Ancellet G. and F. Ravetta. 1998. Appl. Opt., 37, 24, 5509-5521.

[22] Froidevaux, M., et al. 2013. A Raman lidar to measure water vapor in the atmospheric boundary layer. Advances in Water Resources, 51, 345-356. DOI: 10.1016/j.advwatres.2012.04.008.

[23] Arshinov, Y. F., et al. 2003. Proceedings of the ISTP 2003, 142-144.

[24] Lui F. and F. Yi. 2013. Appl. Opt., 52, 28, 6884-6895.

[25] Fochesatto, J., et al. 2004. Advances in Space Research, 34, 10, 2227-2231.

[26] Ristori, P., et al. 2003. Optics and Laser in Engineering, 40, 91-104.

[27] Fochesatto, J., et al. 2005. Proc. of SPIE, 5887, doi: 10.1117/12.620970.

[28] Fochesatto, J., et al. 2008. IJHSES. 18, 3, 713-726. 